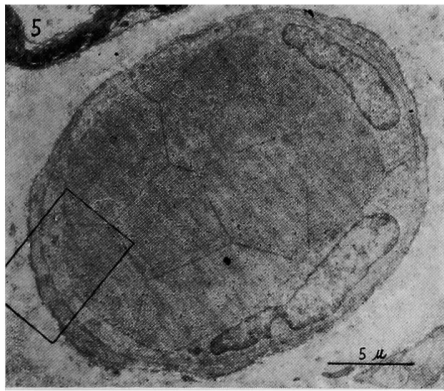

Photo. 5. The rabbit was examined two months after regional cooling of 3 hour duration. $\times 4,000$

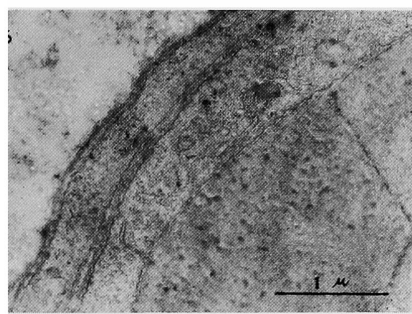

Photo. 6. The higher magnification of Photo 5. $\times 32,500$

\title{
169. The Effect of Regional Cooling on Microcirculation in the Great Occipital Nerve of the Rabbit, A Patho-Dynamic Study
}

\author{
Masashichi Kawano, Shobu Shibata and Hidenobu Oshibuchi \\ 2nd Department of Surgery, Nagasaki University School of Medicine
}

We have been studying the effect of regional cooling of the occipital region upon the great occipital nerve, suspecting that the use of ice pillow in the head injury patints could be one of the causes of post-traumatic chronic headache, at least of neuralgic type with tenderness of the great occipital nerves. In this paper we report on the effect of regional cooling upon microcirculation of the great xccipital nerve.

Arterioles and capillaries in the great occipital nerve of the rabbit were observed for 6 hours under Speroperk microscope during constant regional cooling in which hypodermal temperature was controlled around $5^{\circ} \mathrm{C}$.

In the normal state, erythrocytes are distributed evenly in the arteriole flowing so one direction as a homogenous current, with thick axial stream and thinner narginal stream. In the capillary each erythrocyte runs independently one after another.

At the end of the first cooling hour, vasomotion increases demonstrating nconsistency of the diameter of the wall. Stasis appears and sludge phenomenon Jegins, but they reverse to the normal state, if cooling is interrupted. 


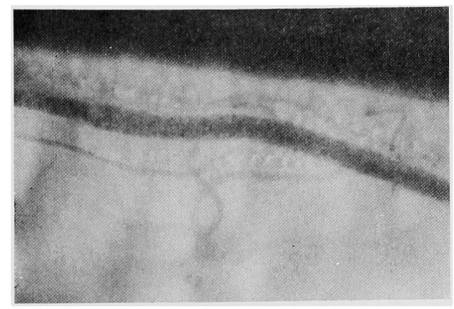

Fig. 1. In the normal state. $\times 60$

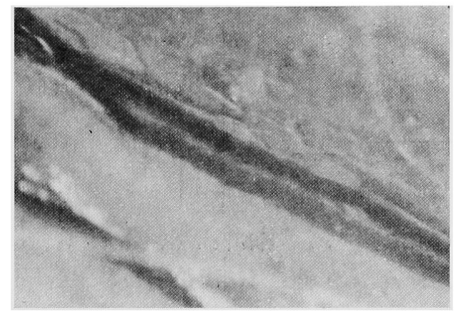

Fig. 3. After 3 hours of cooling. $\times 60$

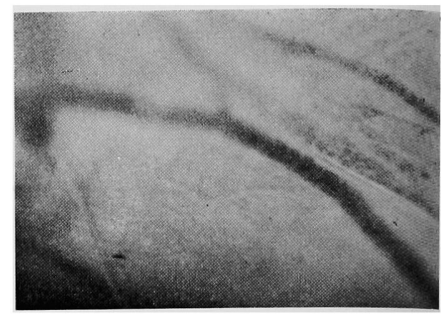

Fig. 2. At the end of the first cooling hour. $\times 60$

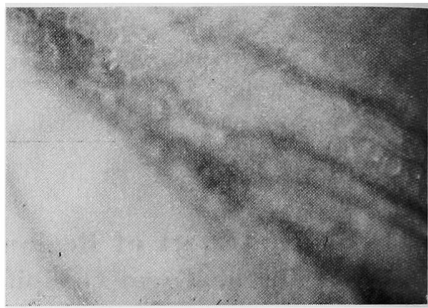

Fig. 4. After 6 hours or longer cooling $\times 60$

After 3 hours of cooling, the vessels dilate unevenly. Sludge phenomenon advances. The leukocytes tend to stick to the vessel wall. The phenomenon called plasma skimming occurs at the bifurcation of the arteriole. In the true capillaries erythrocytes run intermeittently with interval of vacancy. Cessation of cooling does not reverse all these phenomena, but leaves some slowing of the current in the capillaries.

After 6 hours or longer cooling, sludge phenomenon becomes distinct, forming thrombus mass of erythrocytes and leukocytes. Finally rupture of the vessel wall and leakage of the blood begin. Cessation of cooling no longer reverse these conditions.

These findings suggest that the habit of cooling the head with ice bag or pillow long after injury disturbs microcirculation in the great occipital nerve leading to chronic neuralgic headache with increased irritability and lowered pain threshold of the nerve as the result of hypoxia. 